\title{
Determination of electron beam parameters by means of laser-Compton scattering
}

\author{
K. Chouffani, ${ }^{1, *}$ F. Harmon, ${ }^{1}$ D. Wells, ${ }^{1}$ J. Jones, ${ }^{2}$ and G. Lancaster ${ }^{2}$ \\ ${ }^{1}$ Idaho Accelerator Center, 1500 Alvin Riken Drive, Pocatello Idaho 83209, USA \\ ${ }^{2}$ Idaho National Engineering and Environmental Laboratory, P.O. Box 1625, MS 2802, Idaho Falls, Idaho 83415-2802, USA
}

(Received 13 January 2006; published 2 May 2006)

\begin{abstract}
Laser-Compton scattering (LCS) experiments were carried out at the Idaho Accelerator Center using the $5 \mathrm{~ns}$ (FWHM) and $22 \mathrm{MeV}$ electron beam. The electron beam was brought to an approximate head-on collision with a $29 \mathrm{MW}, 7 \mathrm{~ns}$ (FWHM), $10 \mathrm{~Hz} \mathrm{Nd}$ :YAG laser. Clear and narrow x-ray peaks resulting from the interaction of relativistic electrons with the Nd:YAG laser second harmonic line at $532 \mathrm{~nm}$ were observed. We have developed a relatively new method of using LCS as a nonintercepting electron beam monitor. Our method focused on the variation of the shape of the LCS spectrum rather than the LCS intensity as a function of the observation angle in order to extract the electron beam parameters at the interaction region. The electron beam parameters were determined by making simultaneous fits to spectra taken across the LCS x-ray cone. This scan method allowed us also to determine the variation of LCS xray peak energies and spectral widths as a function of the detector angles. Experimental data show that in addition to being viewed as a potential bright, tunable, and quasimonochromatic x-ray source, LCS can provide important information on the electron beam pulse length, direction, energy, angular and energy spread. Since the quality of LCS x-ray peaks, such as degree of monochromaticity, peak energy and flux, depends strongly on the electron beam parameters, LCS can therefore be viewed as an important nondestructive tool for electron beam diagnostics.
\end{abstract}

DOI: 10.1103/PhysRevSTAB.9.050701

PACS numbers: 13.60.Fz, 41.75.Ht, 29.30.Kv, 07.85.Fv

\section{INTRODUCTION}

Laser-Compton scattering (LCS) $\mathrm{x}$ rays are generated when a high-energy electron beam interacts with laser photons; the electrons scatter the low energy photons to higher energy at the expense of the electrons kinetic energy. LCS radiation can also be viewed as the radiation generated by electrons oscillating in a laser electric field, making LCS mechanism similar to that of channeling radiation [1]. The radiation is emitted in the direction of the electron beam, in a cone of half angle equal to $1 / \gamma$ where $\gamma$ is the electron relativistic factor. The energy of LCS $x$ rays is tunable [2], the $\mathrm{x}$-ray energy can be tuned by changing the electron beam energy, laser wavelength, observation and crossing angles with respect to the electron beam direction. LCS can happen at any crossing angle (Fig. 1), the most common interaction geometries are the $90^{\circ}$ geometry, where the electron beam is perpendicular to the laser beam and $180^{\circ}$ geometry, where the electron beam collides head-on with the laser beam. In the $180^{\circ}$ geometry, the $\mathrm{x}$-ray pulse length is primarily determined by the electron beam pulse length [3], and in the $90^{\circ}$ configuration, the $\mathrm{x}$-ray pulse length is given by the transit time of the laser across the electron beam [4]. Though LCS has been proven to be a useful x-ray source for medical imaging [5], the properties LCS $x$ rays are strongly dependent on the electron beam parameters, and therefore LCS can be viewed as a nonintercepting electron beam monitor [6,7].

*Email address: khalid@athena.physics.isu.edu
The goal of this experiment described in this paper is to use LCS as a nondestructive electron beam diagnostic tool in order determine several electron beam parameters such as the electron beam energy, energy spread, beam divergence, and electron beam direction. The electron beam parameters are determined by performing spatial scans across the LCS $x$-ray cone. The variation of the shape of the LCS spectrum for different $\mathrm{x}$-ray detector positions provides information on the electron beam parameters mentioned above.

An important application is a transverse phase space mapping of the electron beam profile [8]. For an electron beam spot size much larger than that of the laser at the

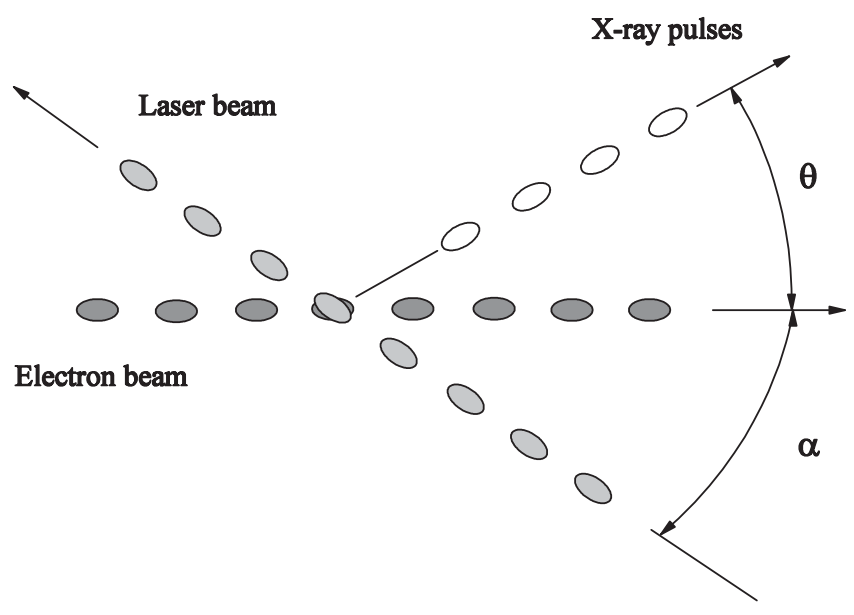

FIG. 1. LCS interaction geometry, $\alpha$ is the crossing angle, and $\theta$ is the emission angle. 


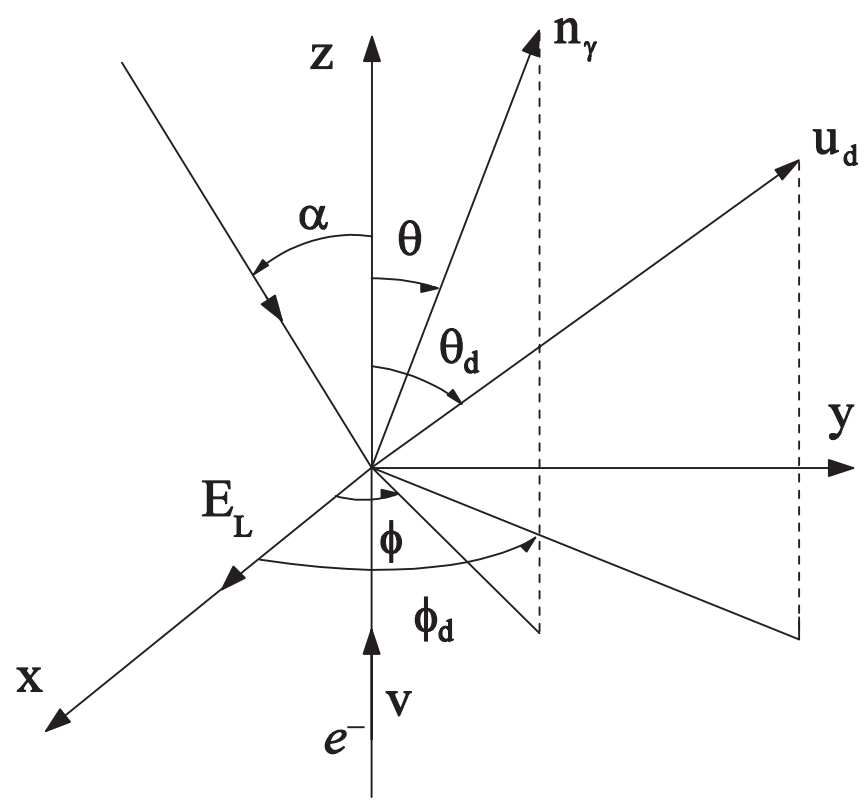

FIG. 2. Orientation in the case of a collision in the $y-z$ plane. The $\mathbf{x}$ and $\mathbf{y}$ axes will be referred to as the vertical and horizontal axis, respectively. $\mathbf{n}_{\gamma}$ and $\mathbf{u}_{d}$ are the x-ray and detector directions, respectively. $(\theta, \phi)$ and $\left(\theta_{d}, \phi_{d}\right)$ are the $\mathrm{x}$-ray and detector angles, respectively. $(\theta, \phi)$ and $\left(\theta_{d}, \phi_{d}\right)$ are the x-ray and detector angles, respectively, and $\mathbf{E}_{\mathbf{L}}$ is the laser polarization direction.

interaction region, it is possible, by scanning the focused laser beam across the electron beam, to determine the beam emittance as well as other electron beam parameters at every point of the electron beam transverse profile.

Because this diagnostic method uses LCS spectra collected over several electron-laser pulse collisions and detector positions, the measured parameters of the electron beam are subject to instabilities in the accelerator and electron beam delivery system and therefore do not allow accurate characterization of the electron beam bunch parameters.

\section{THEORETICAL STUDY OF LASER-COMPTON SCATTERING}

LCS x-ray energy in the laboratory frame (Fig. 2) can be determined from the conservation of energy and momentum and is given by

$$
\begin{aligned}
E_{\gamma} & =\frac{E_{L}(1+\beta \cos \alpha)}{1-\beta \cos \theta+\frac{E_{L}}{E_{B}}(1+\cos \theta \cos \alpha-\sin \theta \sin \phi \sin \alpha)} \\
& \approx \frac{E_{L}(1+\beta \cos \alpha)}{1-\beta \cos \theta},
\end{aligned}
$$

where $E_{L}$ is the laser photon energy, $\beta=v / c$ is the ratio of the electron and light velocities, $\alpha$ is the crossing angle, $\theta$ and $\varphi$ are the emission and azimuthal angles, respectively, and $E_{B}$ is the electron beam total energy. For collision geometries where $\alpha \approx 0$ and for emission angles close to the electron beam direction $(\sin \theta \approx \theta)$, Eq. (1) reduces to $E_{\gamma} \approx E_{M} /\left(1+\gamma^{2} \theta^{2}\right)$, where $E_{M}=4 \gamma^{2} E_{L}$ is the maximum energy generated in the forward direction, i.e., electron beam direction, for a head-on collision. The maximum LCS radiation energy is twice Doppler shifted upward from the incident laser photon energy.

The LCS energy spectrum for head-on collision $(\alpha=0)$ is derived from the expression of the differential Compton cross section in the laboratory frame [9]. Taking into account the electron beam angular spread and energy deviation and assuming that the contributions of the laser frequency bandwidth and the finite interaction length to the LCS spectrum are negligible compared to those of the electron beam energy and angular deviations, the LCS spectrum generated by the collision of single electron and laser bunches, and for a linearly polarized laser beam, can be written as

$$
\frac{d N_{\gamma}}{d E_{\gamma}}=\int_{d \Omega_{d}} \frac{d N_{\gamma}}{d \Omega_{d} d E_{\gamma}} d \Omega_{d}
$$

where $d \Omega_{d}=d \theta_{x, d} d \theta_{y, d}$ is the detector solid angle, $\theta_{x, d} \approx$ $\theta_{d} \cos \varphi_{d}, \theta_{y, d} \approx \theta_{d} \sin \varphi_{d}$, and

$$
\begin{aligned}
\frac{d N_{\gamma}}{d \theta_{x, d} d \theta_{y, d} d E_{\gamma}}= & L F\left(E_{\gamma}\right) \frac{d \sigma}{d \theta_{x, d} d \theta_{y, d} d E_{\gamma}} \\
= & \frac{L F\left(E_{\gamma}\right) 2 r_{0}^{2}}{(2 \pi)^{3 / 2} \sigma_{x} \sigma_{y} \sigma_{e}} \int_{0}^{\infty} \int_{0}^{2 \pi} \frac{(1+\beta)}{E_{M}}\left[(\cos 2 \phi+1) \frac{E_{\gamma}}{E_{M}}\left(\frac{E_{\gamma}}{E_{M}}-1\right)+\frac{1}{2}\right] \\
& \times \exp \left(-\frac{\left(\gamma^{\prime}-\gamma\right)^{2}}{2 \sigma_{e}^{2}}\right) \exp \left(-\frac{\left(\theta_{x, d}-\theta_{x}\right)^{2}}{2 \sigma_{x}^{2}}\right) \exp \left(-\frac{\left(\theta_{y, d}-\theta_{y}\right)^{2}}{2 \sigma_{y}^{2}}\right) d \phi d \gamma^{\prime}
\end{aligned}
$$

where $r_{0}$ is the electron radius, $E_{M}=4 \gamma^{\prime 2} E_{L}, \theta_{x} \approx$ $\theta \cos \phi$ $\theta_{y} \approx \theta \sin \phi$ and $\theta \approx \sqrt{1-E_{M} / E_{\gamma}} / \gamma^{\prime}$.

The $x$ and $y$ subscripts correspond to directions along the $\mathbf{x}$ and $\mathbf{y}$ directions. $\varphi$ is the azimuthal angle with respect to the laser polarization direction. $\sigma_{x}$ and $\sigma_{y}$ are the vertical and horizontal rms widths of the electron beam angular distribution, respectively, and $\sigma_{e}$ is the rms widths of the electron beam energy distribution. $L$ is the single collision luminosity (see below) and $F\left(E_{\gamma}\right)$ takes into account the $\mathrm{x}$-ray absorption in the different media. In order to include the detector energy resolution and efficiency, Eq. (3) must 
be convoluted with the detector response, and $F\left(E_{\gamma}\right)$ must include the detector efficiency.

Equation (3) can similarly be written as

$$
\begin{aligned}
\frac{d N_{\gamma}}{d \theta_{x, d} d \theta_{y, d} d E_{\gamma}} \approx & \frac{L F\left(E_{\gamma}\right) 4 r_{0}^{2}}{(2 \pi)^{3 / 2} \sigma_{x} \sigma_{y} \sigma_{\gamma} E_{M}} \int_{0}^{\infty} \int_{0}^{2 \pi}\left[(\cos 2 \phi+1) \frac{E_{\gamma}^{\prime}}{E_{M}}\left(\frac{E_{\gamma}^{\prime}}{E_{M}}-1\right)+\frac{1}{2}\right] \times \exp \left(-\frac{\left(E_{\gamma}^{\prime}-E_{\gamma}\right)^{2}}{2 \sigma_{\gamma}^{2}}\right) \\
& \times \exp \left(-\frac{\left(\theta_{x, d}-\theta_{x}\right)^{2}}{2 \sigma_{x}^{2}}\right) \exp \left(-\frac{\left(\theta_{y, d}-\theta_{y}\right)^{2}}{2 \sigma_{y}^{2}}\right) d \phi d E_{\gamma}^{\prime} .
\end{aligned}
$$

where $E_{M}=4 \gamma^{2} E_{L}, \quad \theta \approx \sqrt{1-E_{M} / E_{\gamma}^{\prime}} / \gamma, \quad$ and $\sigma_{\gamma} \approx 2 E_{M} \sigma_{e} /\left(\gamma\left(1+\gamma^{2} \theta_{d}^{2}\right)^{2}\right)$ is the rms x-ray energy spread resulting from the electron beam energy deviation [Eq. (1)].

We have assumed in Eqs. (3) and ( $\left.3^{\prime}\right)$ that the electron beam angular and energy distributions are Gaussians. The contribution of the laser frequency bandwidth can be included by convoluting Eqs. (3) and (3') with the laser photon energy distribution.

The general expression of the single collision luminosity can be found in [10]. In the case of a head-on collision between the electron and laser pulses the luminosity is given by $L=N_{e} N_{L} /\left(2 \pi \sqrt{x_{\sigma}^{2}+\sigma_{w}^{2}} \sqrt{y_{\sigma}^{2}+\sigma_{w}^{2}}\right)$, where $N_{e}$ and $N_{L}$ are the numbers of electrons and photons in the electron and laser bursts, respectively. $x_{\sigma}$ and $y_{\sigma}$ are the rms widths of the electron beam spatial distributions along the transverse $\mathbf{x}$ and $\mathbf{y}$ directions, respectively. $\sigma_{w}$ is the rms width of the laser beam transverse spatial distribution. As a reminder, $\sigma_{w}$ is equal to $0.5 w$, where $w$ is the laser waist. For small crossing angles ( $\sin \alpha \approx \alpha$ ), for example, in the $y-z$ plane, the single collision luminosity is given by $L \approx N_{e} N_{L} /\left(2 \pi \sqrt{x_{\sigma}^{2}+\sigma_{w}^{2}} \sqrt{y_{\sigma}^{2}+\sigma_{w}^{2}+\alpha^{2}\left(z_{\sigma}^{2}+\sigma_{L}^{2}\right) / 4}\right)$, where $z_{\sigma}$ and $\sigma_{L}$ are the rms widths of the electron and laser beam longitudinal distributions.

For an ideal electron beam with no energy or angular spread, and a monochromatic laser pulse and for an infinitely small solid angle, the LCS spectrum is practically a delta function. Several parameters contribute to the width and the peak energy of the LCS spectrum; among those are the electron beam angular and energy spread, laser wavelength and frequency bandwidth, detector angle with respect to the electron beam direction, and detector solid angle. As can be seen from Eq. (1), electrons making an angle $\sigma$ with the forward direction will emit x rays with energies lower than those emitted by electrons along the forward direction. This will cause the LCS to broaden and acquire a low energy tail. Similarly, an observation angle away from the forward direction will collect $\mathrm{x}$ rays emitted by electron traveling with different directions with respect to interaction point-detector axis. The spectral broadening will increase as the observation angle is increased due to larger emission angles. Detector collimation is also an important factor in reducing the width of LCS spectra since it enables the detector to collect only $\mathrm{x}$ rays within a certain solid angle. There exist however a minimum collimator diameter below which the shape of the LCS spectrum does not change. The dependence of LCS spectral width on the electron beam energy spread, and the laser linewidth is obvious according to Eq. (1). For an observation angle in the forward direction, the spectral broadening $\Gamma_{\mathrm{FWHM}}$ resulting from each contribution is equal to $2 E_{M} \Gamma_{\gamma} / \gamma$ and $E_{M} \Gamma_{L} / E_{L}$, respectively, where $\Gamma_{\gamma}$ and $\Gamma_{L}$ are the FWHM of the electron beam and laser photon energy distributions, respectively. The finite interaction length contributes also to the width of the LCS spectrum, with a spectral broadening equal to $\Gamma_{I L}=(1+\beta)^{2} \gamma^{2} h c / l$, where $l$ is the interaction length and $h$ the Planck's constant. For an electron beam with no angular spread, the LCS spectrum FWHM is obtained as the quadrature summation of the different broadening factors mentioned above. Because the electron beam divergence causes the LCS spectrum to have an asymmetric shape (low energy tail) with respect to the energy of the peak, the FWHM of the spectrum cannot be given simply by the quadrature sum of the different line broadening mechanisms, but must be determined directly from the LCS spectrum [Eq. (2)]. The laser wavelength and electron beam energy affect the width of the LCS spectrum as well. LCS spectra generated using the fundamental laser wavelength are narrower than those using laser harmonics.

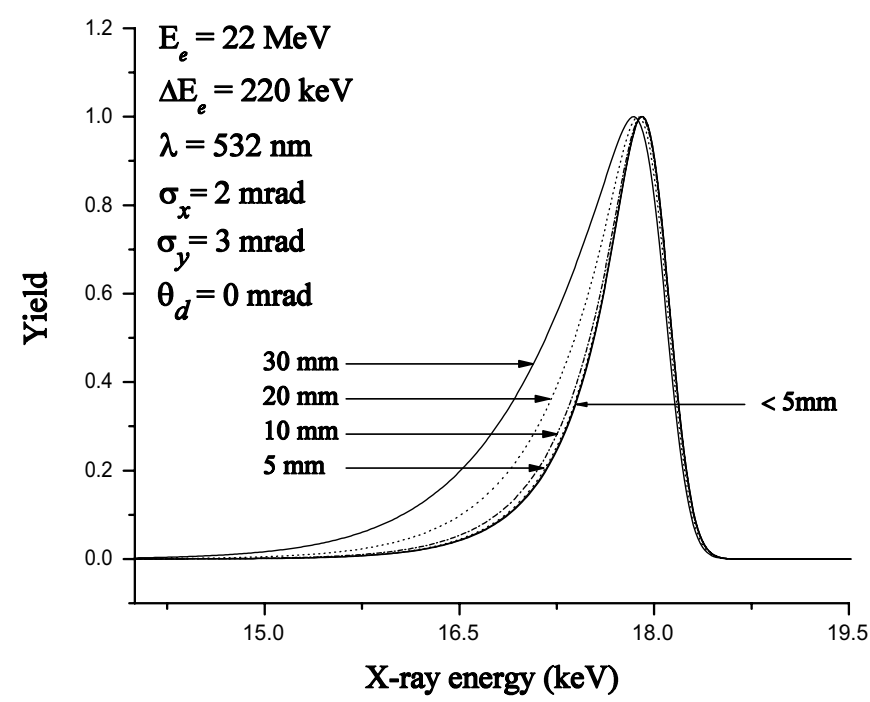

FIG. 3. Variation of amplitude normalized LCS spectrum as a function of the collimator size. The detection angle was equal to $0 \mathrm{mrad}$ and the distance between the interaction point and collimator was equal to $6 \mathrm{~m}$. Simulations show that the shapes of the spectra do not change when the collimator radius is less than $5 \mathrm{~mm}$. 

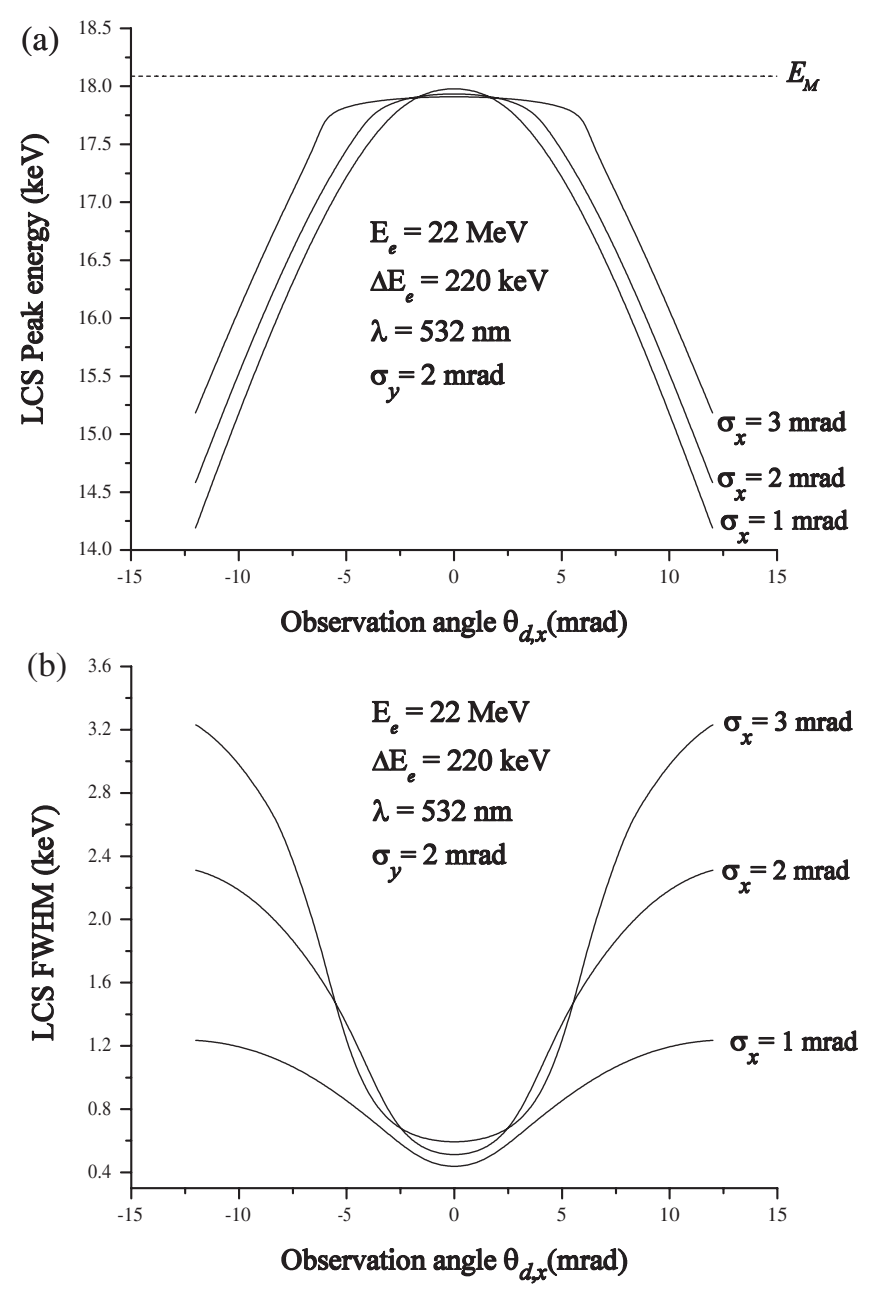

FIG. 4. (a) Variation of LCS peak energy and (b) FWHM as a function of detector angle $\theta_{d, x}$ with respect to the forward direction for different values of $\sigma_{x}$. The angular scan is along the laser electric field direction (vertical direction, i.e., $\theta_{d, y}=$ $0 \mathrm{mrad}$ ). In (a) the angles at which the energy curves intersect are roughly equal to $\left(\sigma_{x, 1}+\sigma_{x, 2}\right) / 2$, where $\sigma_{x, 1}$ and $\sigma_{x, 2}$ are 2 different vertical rms widths of the electron beam angular distribution. The dotted line corresponds to the value of the maximum x-ray energy $E_{M}=18.09 \mathrm{keV}$. The curves were generated using Eq. (3).

As mentioned above, for an ideal electron beam and laser, the LCS spectrum is a delta function located at the maximum energy $E_{M}$ (when the observation angle is along the electron beam direction), the electron beam divergence and energy spread and the laser frequency bandwidth will cause the energy of the LCS peak to shift to lower energies (see below).

Angular scans across the LCS x-ray cone can give information on the electron beam direction with respect to the interaction point-detector direction, electron beam angular spread, energy, and energy deviation.

In the following simulations, we have neglected the contribution of the laser frequency bandwidth as well as the broadening due to the finite interaction length. Figure 3
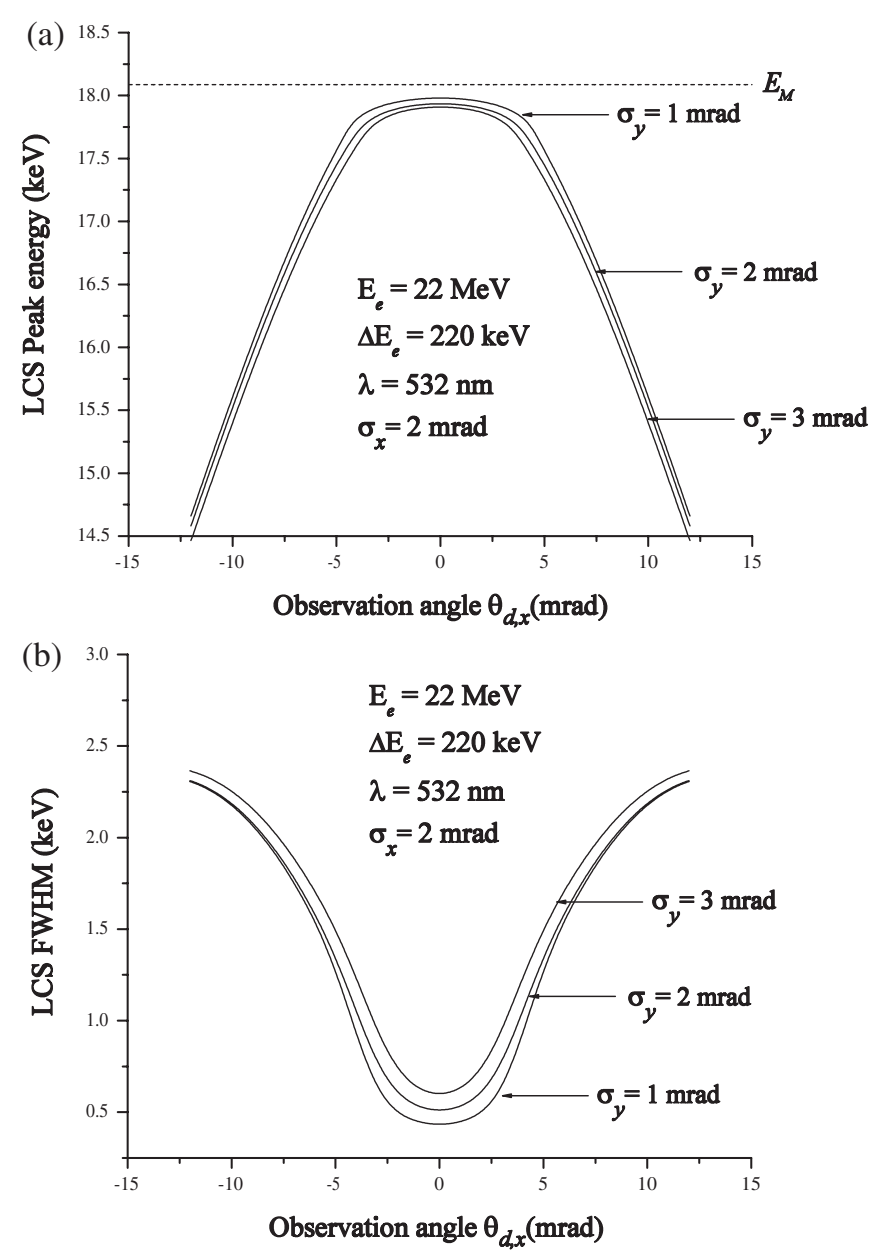

FIG. 5. Same as Figs. 4(a) and 4(b) but for different values of $\sigma_{y}$.

shows the different shapes of the amplitude normalized LCS spectrum for different values of the detector collimator radius and for a distance between the detector and interaction point equal to $6 \mathrm{~m}$. The spectra were generated using Eq. (3) with $\theta_{d}=0$ and for a given set of electron beam parameters. The simulations show that the shape of the spectra does not change when the detector collimator radius is less than $5 \mathrm{~mm}$. A similar result was obtained for a detector at an arbitrary position along the vertical axis, away from the forward direction.

Figures 4 and 5 show the variation of (a) the LCS x-ray peak energy and (b) FWHM as a function of the detector angle $\theta_{d, x}$ along the vertical direction $\left(\theta_{d, y}=0\right)$ in the case of head-on collision for different values of the electron beam vertical angular spread and horizontal angular spread, respectively. As expected the effect of the electron beam vertical angular divergence $\sigma_{x}$ is more pronounced on the FWHM and the energy of the spectra than that of the horizontal angular spread $\sigma_{y}$. Furthermore the FWHM of the spectra appears to be more sensitive to the changes of $\sigma_{x}$ than the energy of the LCS peak does. For example in Fig. 4, for $\theta_{d, x}=0 \mathrm{mrad}$, 
$\Delta\left[E_{\gamma}\left(\sigma_{x}=2 \mathrm{mrad}\right)-E_{\gamma}\left(\sigma_{x}=1 \mathrm{mrad}\right)\right] / E_{\gamma}\left(\sigma_{x}=\right.$ $2 \mathrm{mrad}) \approx 0.2 \% \quad$ while $\quad \Delta\left[\Delta E_{\gamma}\left(\sigma_{x}=2 \mathrm{mrad}\right)-\right.$ $\left.\Delta E_{\gamma}\left(\sigma_{x}=1 \mathrm{mrad}\right)\right] / \Delta E_{\gamma}\left(\sigma_{x}=2 \mathrm{mrad}\right) \approx 14.5 \%$, while for $\quad \theta_{d, x}=5 \mathrm{mrad} \Delta\left[E_{\gamma}\left(\sigma_{x}=2 \mathrm{mrad}\right)-E_{\gamma}\left(\sigma_{x}=\right.\right.$ $1 \mathrm{mrad})] / E_{\gamma}\left(\sigma_{x}=2 \mathrm{mrad}\right) \approx 1.3 \%$ while $\Delta\left[\Delta E_{\gamma}\left(\sigma_{x}=\right.\right.$ $\left.2 \mathrm{mrad})-\Delta E_{\gamma}\left(\sigma_{x}=1 \mathrm{mrad}\right)\right] / \Delta E_{\gamma}\left(\sigma_{x}=2 \mathrm{mrad}\right) \approx$ $36 \%$. Similar curves can be obtained by varying the electron energy spread for given values of the electron beam angular divergence and energy [6].

\section{EXPERIMENTAL RESULTS AND DISCUSSIONS}

The LCS experiment was carried out at the IAC using the $5 \mathrm{~ns}, 22 \mathrm{MeV}$ electron beam from the linear accelerator (LINAC) and the $7 \mathrm{~ns}, 29 \mathrm{MW}$ peak power, $\mathrm{TEM}_{00}$ $\mathrm{Nd}$ :YAG laser. During this experiment, the $532 \mathrm{~nm}$ wavelength was selected by placing a dichroic mirror at the exit of the harmonic generator and steering the $1064 \mathrm{~nm}$ laser beam toward a laser beam dump. At the wavelength of interest, the pulse energy was equal to $0.2 \mathrm{~J}$ and the laser polarization vector was parallel to the $\mathbf{x}$ axis (Fig. 6). The accelerator layout and characteristics can be found in [2]. The layout of the experiment is shown in Fig. 6. The electron beam from the LINAC was bent into the $90^{\circ}$ beam line by a set of four $22.5^{\circ}$ bending magnets toward the experimental area. The electron beam was then focused at the first (upstream) screen where it collided with the laser beam. A slit placed between the $22.5^{\circ}$ bending magnets enabled us to control the electron beam energy distribution, current, and bremsstrahlung background. The electron beam energy spread, for a given electron beam energy, was determined from the slit meter (SM) readout using a simulated calibration curve. Each SM readout gives $\Delta E_{e} / E_{e}$ where $E_{e}$ is the electron beam energy and $\Delta E_{e}$ is presumably the FWHM of the electron beam energy distribution. The slit is closed when the SM value is equal to 55 and the maximum width is reached when the SM value is equal to 1000 . The electron beam current remains constant when the SM readout is equal or greater than 700 .
Most of the LCS spectra were collected for SM readouts $\leq 200$ where the electron beam diameter at the center of the first flag was $\leq 3 \mathrm{~mm}$. After interaction with the laser, the electron beam was then bent by a $45^{\circ}$ bending magnet. The laser together with the optical layout and $\mathrm{x}$-ray detectors were located in a separate room. The laser beam was first expanded and then focused by a $5 \mathrm{~m}$ focal length lens and steered by an off axis mirror toward the center of the center first screen. The distance between the center of the cross containing the focusing lens and the first screen was about $5.5 \mathrm{~m}$, while the distance between the upstream and downstream screens was about $0.38 \mathrm{~m}$.

The angle between the laser and the beam-line axis at the center of the first flag was about $6.2 \mathrm{mrad}$. The estimated laser spot size $2 w$ at the interaction point was equal to $2.5 \mathrm{~mm}$. The repetition rate of the LINAC and laser was equal to $10 \mathrm{~Hz}$. synchronization between the laser and electron beam was achieved by sending two pretrigger TTL signals, preceding the electron gun trigger by $\pm 10 \mu$ s (variable), to the laser flash lamp and $Q$-switch, respectively. For proper laser-electron beam timing and in order to minimize the jitter between the laser and the electron beam, the trigger to the flash lamp was delayed by slightly less than $100 \mathrm{~ms}$ while keeping the delay between the flash lamp and $Q$-switch of the order of $430 \mu \mathrm{s}$. The $Q$-switch was then triggered on the consecutive trigger pulse with respect to the flash lamp trigger.

A photomultiplier (PMT) and a fast pick up current monitor (Fig. 6) were used to monitor the delay between the laser and electron beam, respectively. The LCS x rays traveled through a $50.8 \mu \mathrm{m}$ thick stainless steel (SS) window and were detected using either a high-energy resolution nitrogen cooled $\mathrm{Si}(\mathrm{Li})$ detector or a Peltier cooled PIN detector positioned along the beam-line axis in the laser room. A scintillator detector, placed in the vicinity of the $x$ ray detector, was used to monitor the bremsstrahlung background during electron beam tuning. During the measurements, the electron beam charge per pulse was less or equal $0.7 \mathrm{nC}$. To determine the electron beam energy, the operator measured the $22.5^{\circ}$ bending magnet current and de-

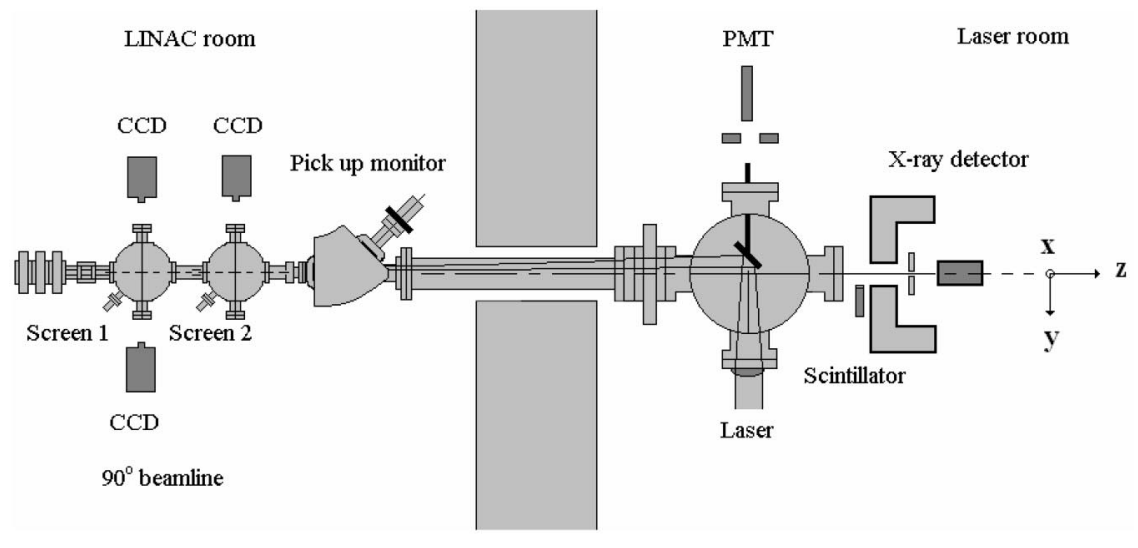

FIG. 6. Top view of experimental setup. The $\mathbf{x}$ axis is normal to the figure and the $\mathbf{z}$ axis is parallel to the beam line. 
ducted the electron beam energy from a theoretical energycurrent conversion table. To align the electron beam along the beam-line axis, the operator tunes the electron beam such that it is as close as possible to the center of each flag while keeping the background level as low as possible. At $22 \mathrm{MeV}$, the electron beam was somewhat unstable and required monitoring. The detectors were calibrated using both calibration sources and bremsstrahlung induced $K_{\alpha}$ lines from $\mathrm{Cu}, \mathrm{Zr}, \mathrm{Mo}$, and $\mathrm{Sn}$. Both calibration methods showed good agreement. Calibration with calibrated sources taken $8 \mathrm{~h}$ apart showed good agreement as well. The detectors energy resolutions were determined with the same methods mentioned above. The Si(Li) and the PIN xray detectors energy resolutions at $18.09 \mathrm{keV}$ were equal to 0.28 and $0.32 \mathrm{KeV}$, respectively. The efficiency of the $\mathrm{Si}(\mathrm{Li})$ detector was close to $100 \%$ in the range of energy of interest and the efficiency of the PIN x-ray detector was equal to $35 \%$ at $18.09 \mathrm{keV}$.

During this experiment the continuum laser was injection seeded, i.e., amplification of single longitudinal mode. Seeding produces a smooth temporal profile and a narrow bandwidth. The injection seeded laser has a frequency bandwidth equal to $90 \mathrm{MHz}\left(\Delta \lambda / \lambda \approx 3.2 \times 10^{-7}\right)$ at $\lambda=$ $1064 \mathrm{~nm}$ and because two $1064 \mathrm{~nm}$ photons are required to generate a $532 \mathrm{~nm}$ photon, the frequency bandwidth of the laser at $\lambda=532 \mathrm{~nm}$ is equal to $127 \mathrm{MHz}(\Delta \lambda / \lambda \approx 2.3 \times$ $10^{-7}$ ). The laser pulse length (FWHM) at $532 \mathrm{~nm}$ when the laser was injection seeded was not measured during this experiment but the value provided by the manufacturer during laser testing was equal to $7.2 \mathrm{~ns}$. The contributions of the laser spectral bandwidth and the finite interaction length to the shapes of the LCS spectra were deemed negligible when compared to the contributions of the electron beam energy spread and divergence and were omitted in the simulations and fits.

Figure 7(a) shows an LCS spectrum recorded by the $\mathrm{Si}(\mathrm{Li})$ detector placed $1.75 \mathrm{~m}$ away from the SS window and $7.43 \mathrm{~m}$ away from the interaction point. The detector subtended a solid angle equal to $0.57 \mu \mathrm{Sr}$. The first peak results from the interaction of the electron beam with the $532 \mathrm{~nm}$ laser line. The additional $\mathrm{x}$-ray peaks are due to pileup, and can be removed by either closing the accelerator slit [Fig. 7(b)], i.e., reducing the electron beam current or by placing an absorber. During these measurements the SM values were 150 and 90 corresponding to macropulse charges of 0.5 and $0.2 \mathrm{nC}$, respectively. Both spectra were collected for $600 \mathrm{~s}$ and at the same delay between the electron beam and laser. The x-ray peak energies and FWHMs were determined from fits to the LCS spectra using the convolution of Eq. (3) with the detector response [Fig. 8(a)]. The fit to the experimental spectra enables us to deduct the LCS spectrum generated inside the vacuum [Fig. 8(b)]. An important observation can be made from Fig. 8(b), one can see that the peak energy is equal to $E_{M}$, which is equal to $18.09 \mathrm{keV}$ for a $22 \mathrm{MeV}$ electron beam.
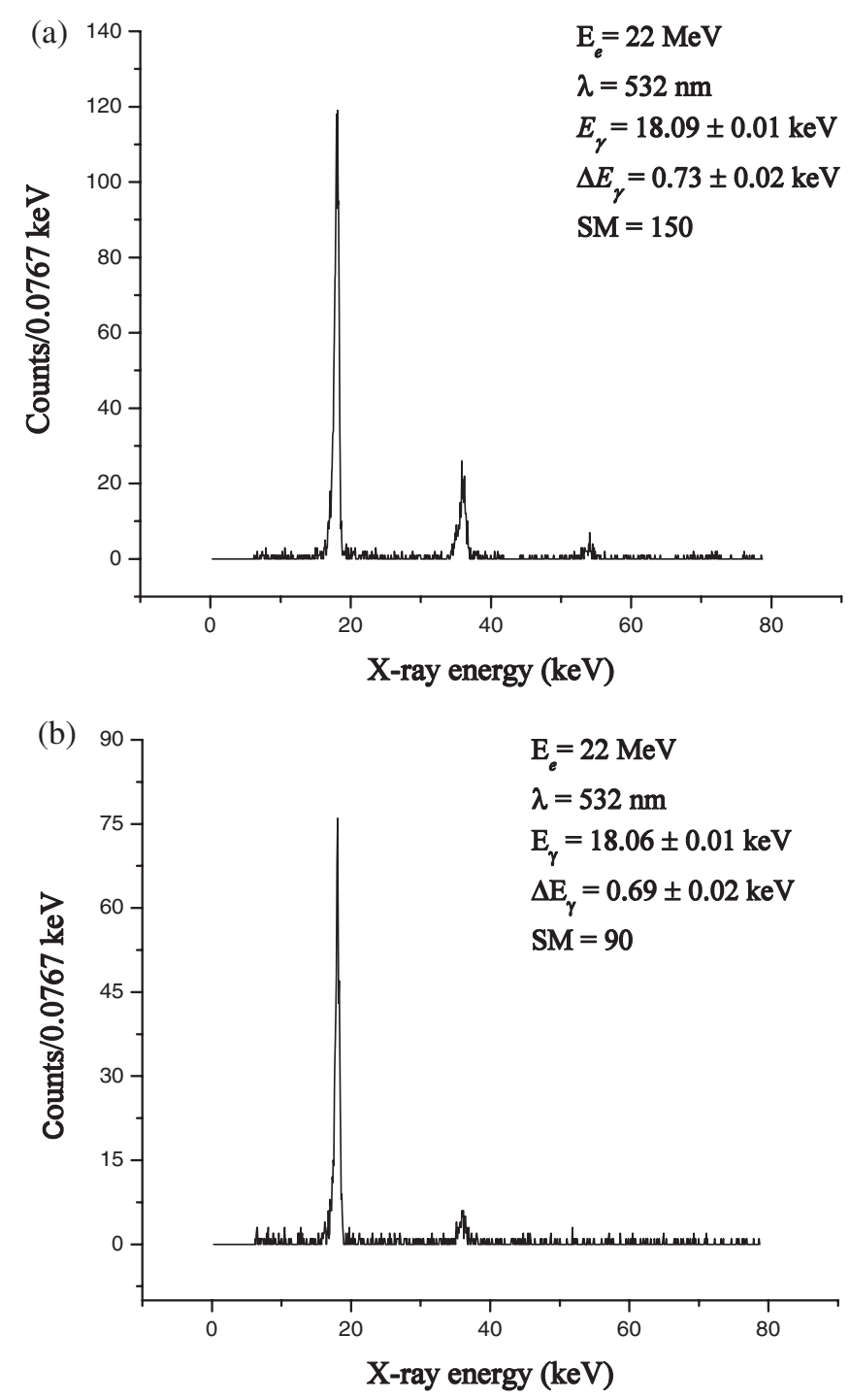

FIG. 7. (a),(b) LCS spectra for a SM readout equal to 150 and 90, respectively. The electron beam macropulse charges were equal to 0.5 and $0.2 \mathrm{nC}$. Only the first pileup peak is visible when $\mathrm{SM}=90$ and the primary LCS peak FWHM is lower than the one at larger slit width.

There are two possibilities where the LCS peak energy can be equal to $E_{M}$. Both cases imply that the electron beam is perfectly aligned with the x-ray detector and that (1) the electron beam has no angular spread, which would mean that the LCS spectrum is a perfect Gaussian whose width is determined by the electron beam energy spread or (2) the electron beam has a nonzero angular spread and a zero energy spread in which case the LCS spectrum will have a sharp vertical edge at $E_{M}$ and a low energy tail. Neither case agrees with the LCS spectrum in Fig. 8(b) since it clearly exhibits an asymmetric shape with a low energy tail, an indication of a nonzero beam divergence, and half a Gaussian like shape above the energy of the peak, which is an indication of a nonzero electron beam energy deviation. This study suggests that the electron beam energy is larger 

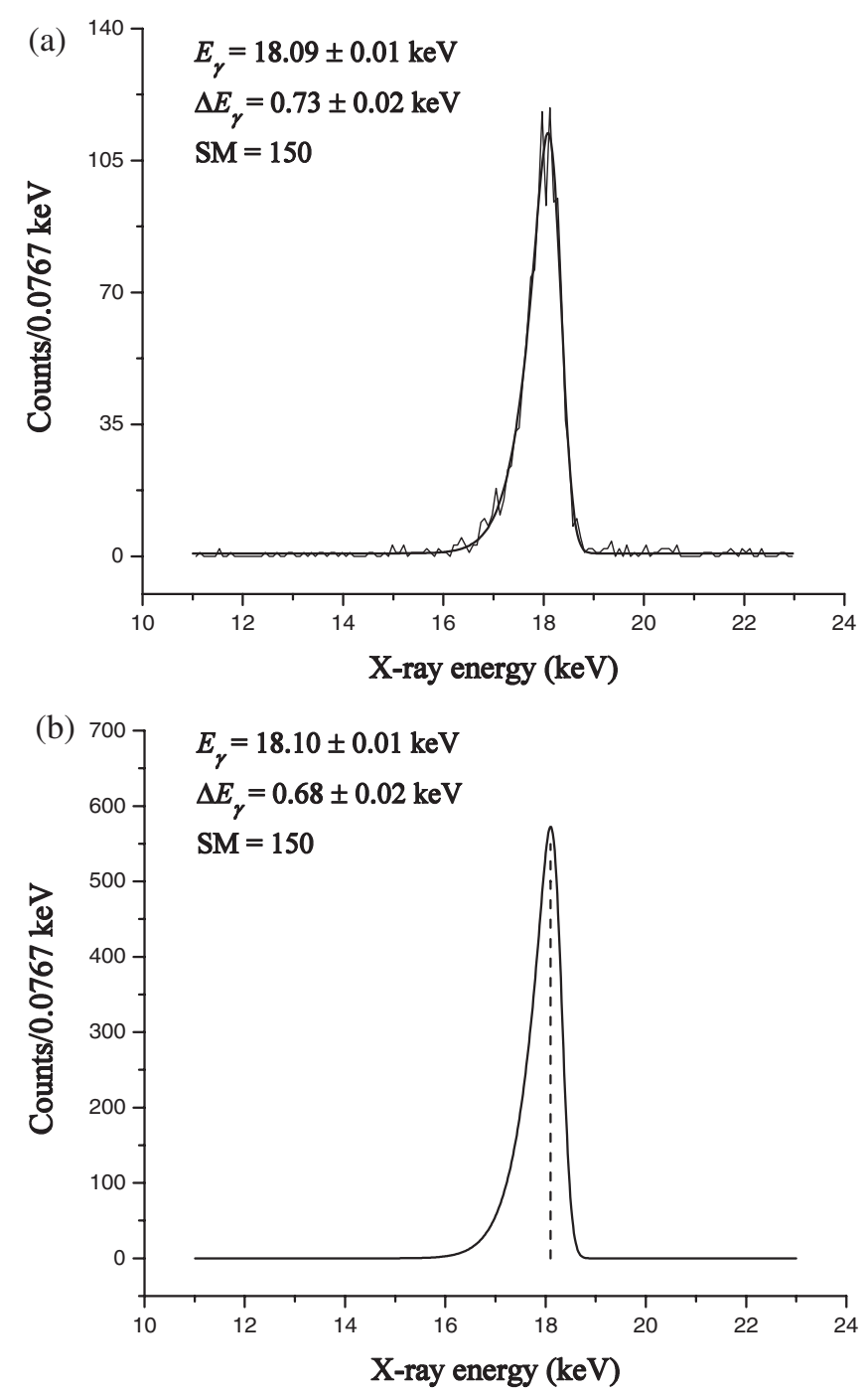

FIG. 8. (a) Fit to the experimental LCS spectrum, for a SM readout equal to 150, using the convolution of Eq. (3) with the detector response. The x-ray absorption in the SS window and air was included in the function $F\left(E_{\gamma}\right)$. (b) LCS spectrum after removing $\mathrm{X}$-ray absorption in different media and deconvolution with detector response. The LCS spectrum was generated using the electron beam parameters from the fit to the experimental spectrum. The vertical dashed line corresponds to the peak energy.

than $22 \mathrm{MeV}$ since for an electron beam with nonzero angular and energy spreads, the energy of the LCS peak is always lower than the maximum energy $E_{M}$ [Figs. 4(a) and 5(a)]. This is a consequence of the convolution of the asymmetric LCS spectrum due to the beam divergence with a symmetric Gaussian distribution representing the electron beam energy deviation.

When the x-ray pulse width is much smaller than the detector resolving time, the detector can record a maximum of only one event per pulse [11]. Pileup occurs when two or more photons are simultaneously registered by the detector. The energy of a pileup $\mathrm{x}$ ray is the sum of the energies of the individual photons collected by the detector. If the pileup is due entirely to LCS x rays and if the electron beam parameters remain constant, the energy distributions of the different order pileup spectra are given by convolutions of the primary LCS peak with itself. Figure 9(a) shows an LCS spectrum taken during a different run after replacing the stainless steel x-ray port window with a $50.8 \mu \mathrm{m}$ thick kapton window. The primary peak is located at $18.16 \pm 0.01 \mathrm{keV}$, the first pileup peak at $36.31 \pm 0.02 \mathrm{keV}$ and the second pileup peak at $54.42 \pm$ $0.03 \mathrm{keV}$. Figure 9 (b) shows $f\left(E_{\gamma}\right)$ the fit to the primary LCS x-ray spectrum. Figure 9(c) shows $g\left(E_{\gamma}\right)=$ $\int f\left(E_{\gamma}^{\prime}\right) f\left(E_{\gamma}-E_{\gamma}^{\prime}\right) d E_{\gamma}^{\prime}$, the convolution of the fit to the primary LCS peak with itself together with the first order pileup peak. Figure $9(d)$ shows the double convolution of the fit to the primary LCS peak with itself $h\left(E_{\gamma}\right)=$ $\int g\left(E_{\gamma}^{\prime}\right) f\left(E_{\gamma}-E_{\gamma}^{\prime}\right) d E_{\gamma}^{\prime}=\iint f\left(E_{\gamma}^{\prime \prime}\right) f\left(E_{\gamma}^{\prime}-E_{\gamma}^{\prime \prime}\right) f\left(E_{\gamma}-\right.$ $\left.E_{\gamma}^{\prime}\right) d E_{\gamma}^{\prime \prime} d E_{\gamma}^{\prime}$ together with the second order pileup peak. The bremsstrahlung levels and peak amplitudes were determined from fits to the individual pileup spectra. Figs. 9(c) and 9(d) show that there is a good agreement between the convolutions and pileup spectra. The shape of a pileup peak should be more sensitive to the electron beam parameters than that of the primary LCS peak and the sensitivity should increase with increasing pileup order.

Before performing the angular scans, we carried out a temporal scan by varying the delay between the laser and electron beam. The temporal scan enabled us to adjust the timing between the laser and electron beam for optimum yield. The accelerator slit width was set at a SM readout equal to 90 in order to minimize the pileup and prevent saturation of the $\mathrm{Si}(\mathrm{Li})$ detector. Figure 10 shows the variation of the LCS intensity as a function of the delay between the laser and electron beam together with the average energy and FWHM of the LCS spectra. The temporal scan gives information on the electron beam longitudinal distribution as well as the jitter between the electron and laser beams. In our current interaction geometry, the temporal scan is also a way of generating x-ray pulses with different pulse lengths without having to change the crossing angle between the laser and electron beam. The maximum pulse length occurs when the interaction length is the longest, i.e., highest intensity. As the delay between the laser and electron beam is varied with respect to the optimum delay, the interaction length becomes smaller resulting in the emission of shorter pulse length $\mathrm{x}$ rays with, as expected, a decrease in the $\mathrm{x}$-ray intensity. The shape of the temporal scan and therefore its width depend on the stability of the electron beam. Even if the electron beam current remains constant, any sudden change in the electron beam direction, electron beam energy or angular spread will affect the LCS intensity and therefore the shape of the temporal scan.

Once the temporal scan was performed we fixed the laser-electron beam delay for optimum yield and installed 

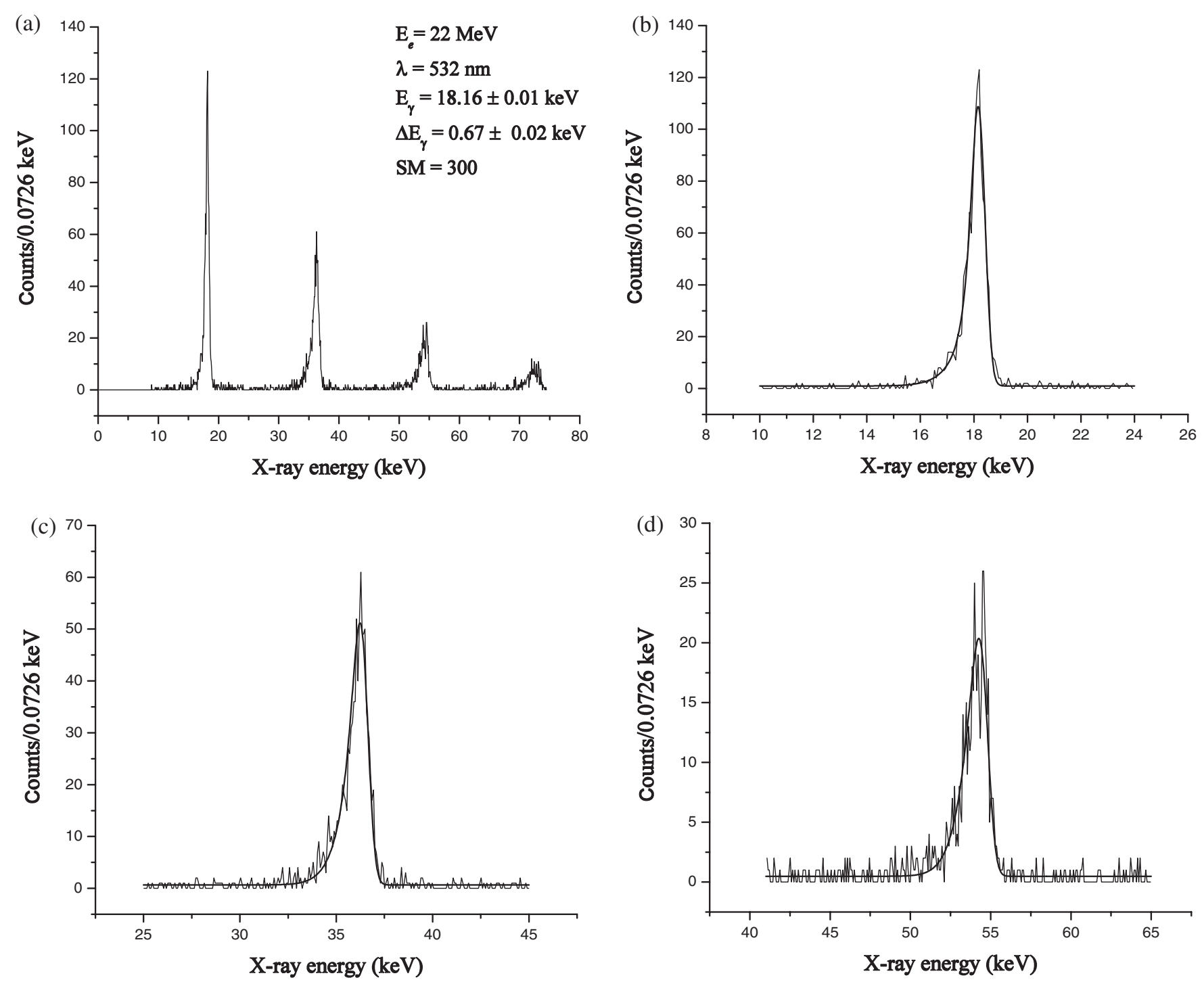

FIG. 9. (a) LCS spectrum taken during a different run at an electron pulse charge equal to 0.9 nC. A $50.8 \mu \mathrm{m}$ kapton window was place in lieu of the $50.8 \mu \mathrm{m}$ SS window. Several pileup peaks are visible due to the low x-ray absorption of kapton. (b) Fit to the LCS primary peak. (c) First pileup peak together with convolution of fit to the primary LCS peak with itself. (d) Second pileup peak together with double convolution of the fit to the primary LCS peak with itself.

the x-ray PIN detector along the beam-line axis. The detector, which was placed on motorized translation stages, was placed $45 \mathrm{~cm}$ away from the SS window and subtended a solid angle equal to $0.2 \mu \mathrm{Sr}$. The distance between the detector and interaction point (center of upstream cross) was equal to $6.13 \mathrm{~m}$. Because of the small solid angle and low detector efficiency for x-ray energies around $18 \mathrm{keV}$ the accelerator slit width was set at a SM readout equal to 200. At this slit width, the charge per macropulse was equal to $0.7 \mathrm{nC}$.

The goal of this experiment is to perform a spatial scan across the x-ray cone in order to determine the electron beam parameters from the set of collected LCS spectra. The center of the x-ray cone corresponds to the LCS spectrum with the highest peak energy and lowest
FWHM, i.e., detection along the electron beam direction (Fig. 4). We first performed a short horizontal scan across the beam-line axis to locate the position of the PIN detector for which the LCS spectrum possesses the highest peak energy and the lowest FWHM in this direction, i.e., smallest observation angle with respect to the electron beam direction. This was done by fitting the LCS spectrum with 2 Gaussians (a broad Gaussian was used to fit the low energy tail) as the scans were carried out. The position of the detector coincided with the beam-line axis. It was not possible to carry out a full horizontal scan because the tip of the $45^{\circ}$ steering mirror (Fig. 6) was close to the beamline axis. The horizontal position of the detector was then fixed and a full vertical scan was performed. We collected 15 spectra during the vertical scan and unlike the method 


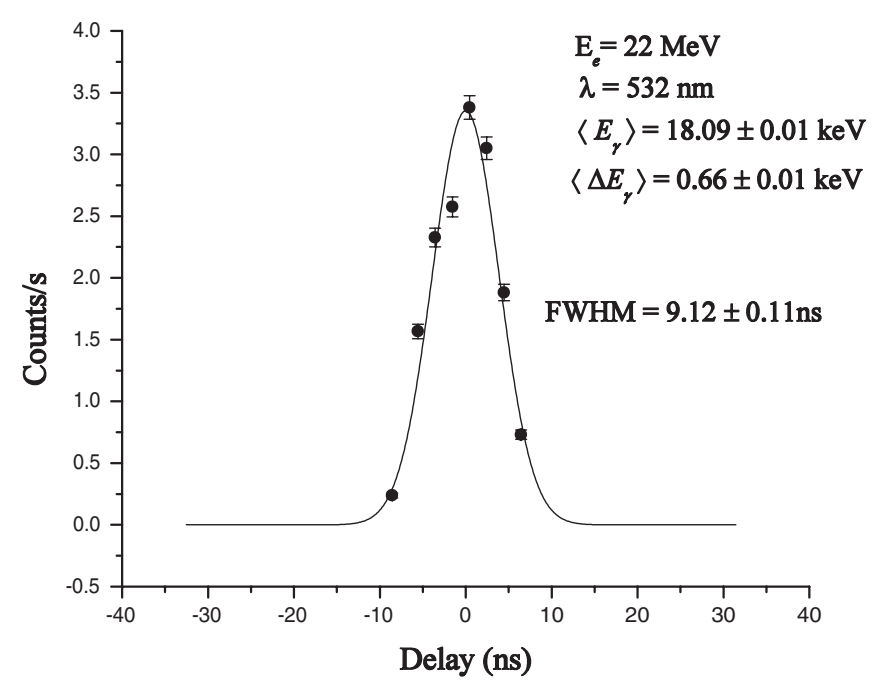

FIG. 10. X-ray intensity as a function of the delay between the laser and electron beam. Each point corresponds to the integrated $\mathrm{x}$-ray yield per second.

mentioned in [4] we used the variation of the shape of the LCS spectrum as a function of the detector position to extract the electron beam parameters. We used the minimization method developed by Box et al. [12,13]. The minimization method determines the common parameters of the fits to several data sets or spectra by minimizing the determinant of the matrix $\left\{v_{i, j}\right\}$ defined as

$$
v_{i, j}=\sum_{k=1}^{n}\left[y_{i}\left(x_{k}\right)-f_{i}\left(x_{k}\right)\right]\left[y_{j}\left(x_{k}\right)-f_{j}\left(x_{k}\right)\right],
$$

where $y_{i}$ and $f_{i}$ correspond to the $i$ th LCS spectrum and fitting function [Eq. (3)], respectively, for a given detector angle and $x_{k}$ is the x-ray energy in the $k$ th energy bin. In the case where there is a single spectrum, i.e., $i=j=1$, Eq. (4) reduces to the well-known expression of the chisquare.

After performing the multiple fits we obtained the values of the following electron beam parameters at the interaction region equal to $E_{e}=22.27 \pm 0.04 \mathrm{MeV}, \Delta E_{e}=$ $0.21 \pm 0.07 \mathrm{MeV}, \sigma_{x}=2.08 \pm 0.13 \mathrm{mrad}, \sigma_{y}=3.05 \pm$ $0.50 \mathrm{mrad}$, and $\theta_{b}=-2.12 \pm 0.32 \mathrm{mrad}$. Where $E_{e}$ is the electron beam energy, $\Delta E_{e}$ is the electron beam energy spread (FWHM), $\sigma_{x}$ and $\sigma_{y}$ are the vertical and horizontal rms widths of the electron beam angular distribution, respectively, and $\theta_{b}$ is the angle between the electron beam direction and beam-line axis.

Figures 11(a) and 11(b) show LCS experimental spectra together with the simulated LCS spectra generated using the electron beam parameters determined from the minimization method for detector vertical angles with respect to the beam-line axis equal to -3.11 and $0.00 \mathrm{mrad}$, respectively. The entire vertical angular range was equal to $14.5 \mathrm{mrad}$, a range that is lower than $1 / \gamma$. The bremsstrahlung levels and peak amplitudes were determined
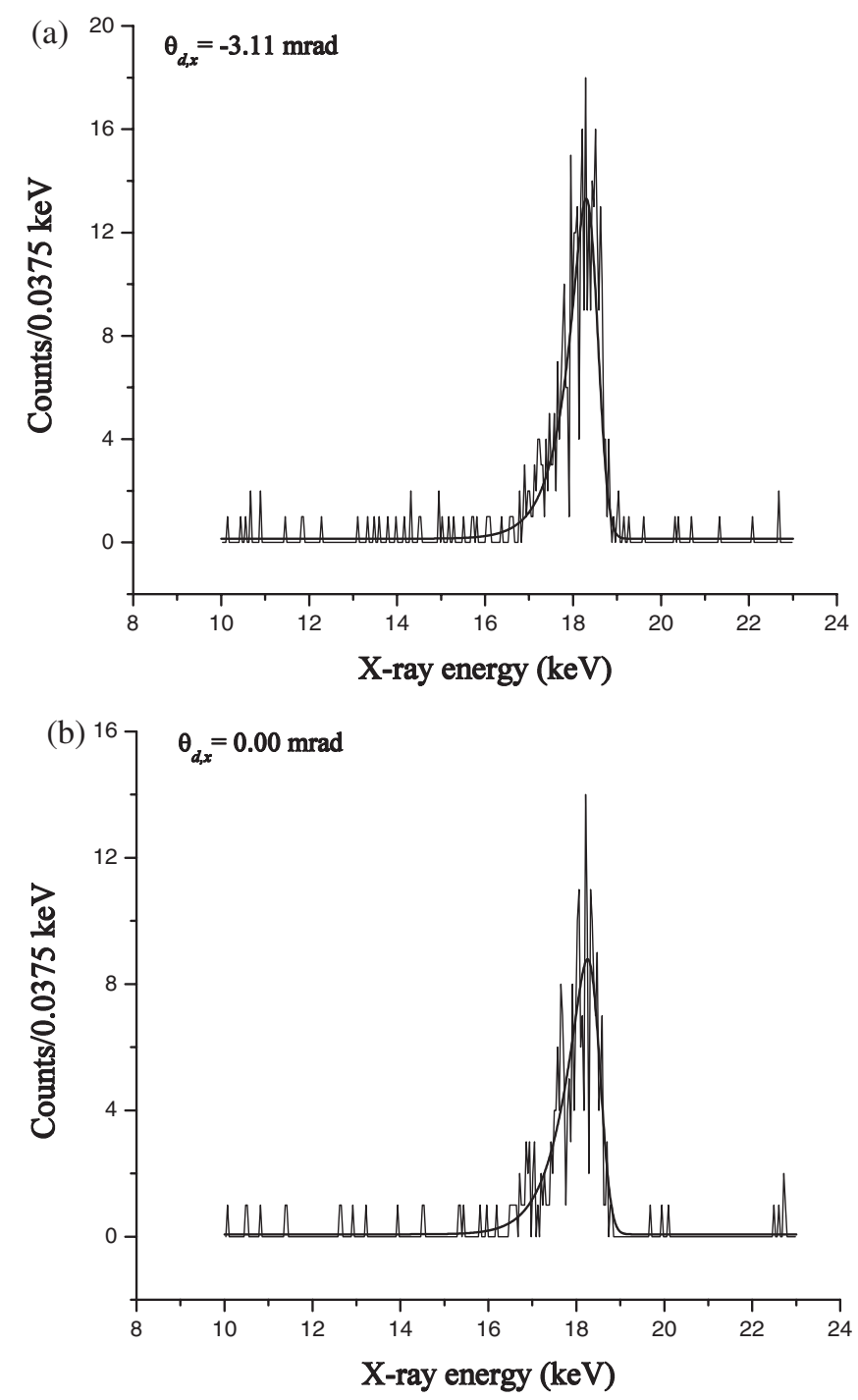

FIG. 11. (a),(b) LCS experimental spectra together with the simulated LCS spectra [Eq. (3)] generated using the electron beam parameters determined from the minimization method for detector vertical angles equal to -3.11 and $0.00 \mathrm{mrad}$, respectively. The $\mathrm{x}$-ray absorption in SS window and air and the detector efficiency were included in the simulations.

from individual fits to the LCS spectra. Figures 12(a) and 12(b) show the variation of the LCS peak energy and FWHM as a function of the detector observation angle, respectively, together with the curves generated using the same parameters. The measured electron beam energy is $1.2 \%$ larger than the one presumed. The difference between $\sigma_{x}$ and $\sigma_{y}$ suggests that the vertical and horizontal focusing focal points may not have been identical. A horizontal scan across the center of the x-ray cone was not performed due to time constraint. This was unfortunate because as can be seen from Fig. 13, the effect of $\sigma_{y}$ on LCS spectra FWHMs along the horizontal direction is more pronounced than the effect on LCS spectra FWHMs along the vertical direction [Fig. 5(b)]. 

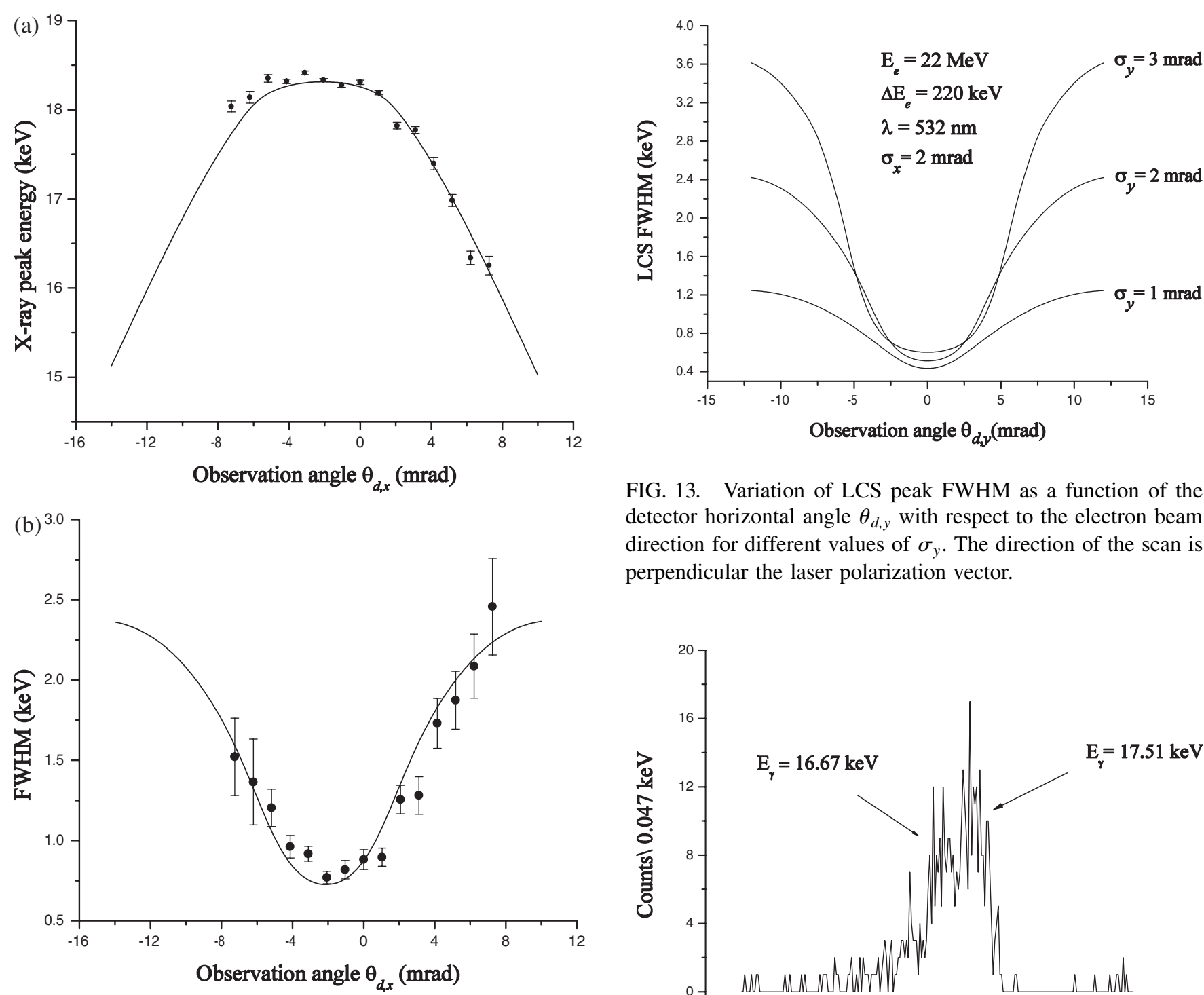

FIG. 13. Variation of LCS peak FWHM as a function of the detector horizontal angle $\theta_{d, y}$ with respect to the electron beam direction for different values of $\sigma_{y}$. The direction of the scan is perpendicular the laser polarization vector.

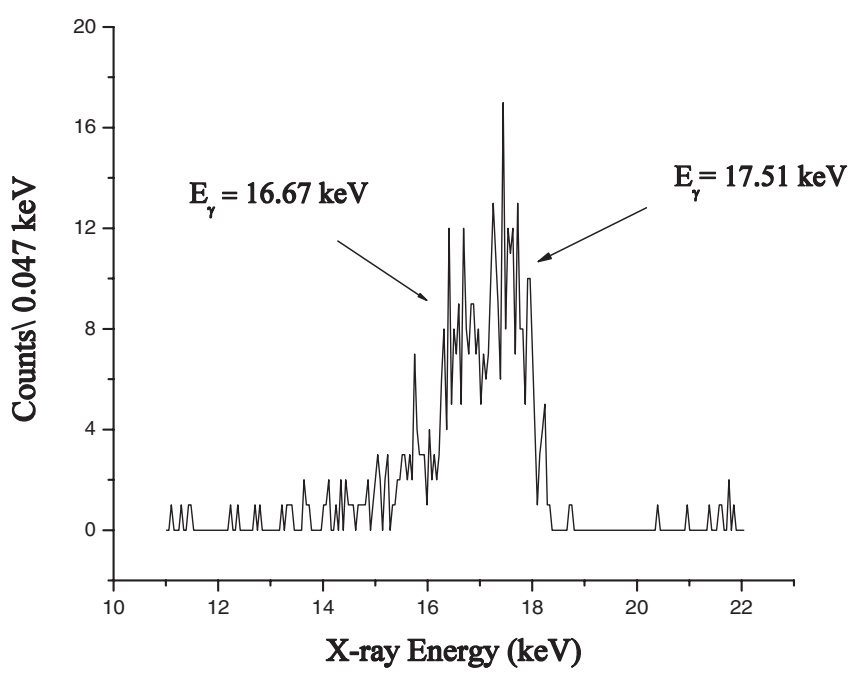

FIG. 12. (a),(b) Variation of LCS x-ray peak energy and
FWHM as a function of the detector vertical angle together with curves generated using the parameters from the multiple fits.

The diagnostic method described above requires a stable electron beam. Instabilities among beam pulses including any spatial jitter or direction drift during data collection will affect the shapes of the LCS spectra (see, for example, Fig. 14), resulting in the measurement of electron beam parameters different from those of the electron beam pulse. During this experiment each spectrum was recorded for $600 \mathrm{~s}$ and no beam tuning was performed during the scans. This experiment could have been carried out in a shorter amount of time if instead of using a single detector to perform the angular scans a matrix of closely spaced detectors placed in front of the x-ray port were available. This would have also minimized the chances of any sudden change in the electron beam parameters during spectra collection.

FIG. 14. LCS spectrum recorded during a different angular scan. The spectrum shows two peaks almost $1 \mathrm{keV}$ apart. The lower peak is suspected to be due a drift in the electron beam direction. The FWHM of the combined peaks is twice as large as that of the higher energy peak.

\section{CONCLUSION}

LCS cannot only be viewed as an intense, tunable and quasimonochromatic $\mathrm{x}$-ray source but can also be viewed as a powerful nonintercepting tool for electron beam diagnostics. Temporal scans give information on the electron beam longitudinal distribution while angular measurements allow the determination of several electron beam parameters as well as the variations of LCS X-ray peak energies and FWHMs across the $\mathrm{x}$-ray cone. This diagnostic method should be compared to other known electron 
beam diagnostic techniques such as optical transition radiation interferometry (OTRI) [14]. Perhaps the most useful application of the method described in this paper is the possibility to determine localized electron beam parameters by scanning a focused laser across the electron beam transverse profile.

\section{ACKNOWLEDGMENTS}

We would like to thank R. B. Fiorito for fruitful discussions. This work was partially funded by the U.S. Department of Energy under DOE Idaho Operations Office Contract No. DE-AC07-99ID13727.

[1] M.Kh. Khokonov and Richard A. Carrigan, Nucl. Instrum. Methods Phys. Res., Sect. B, 145, 133 (1998).

[2] K. Chouffani, D. Wells, F. Harmon, J. Jones, and G. Lancaster, Nucl. Instrum. Methods Phys. Res., Sect. A 495, 95 (2002).

[3] I. V. Pogorelsky, I. Ben-Zvi, T. Hirose, S. Kashiwagi, V. Yakimenko, K. Kusche, P. Siddons, J. Skaritka, T. Kumita, A. Tsunemi, T. Omori, J. Urakawa, M. Washio, K. Yokoya, T. Okugi, Y. Liu, P. He, and D. Cline, Phys. Rev. ST Accel. Beams 3, 090702 (2000).

[4] W. P. Leemans, R. W. Schoenlein, P. Volfbeyn, A. H. Chin, T. E. Glover, P. Balling, M. Zolotorev, K. J. Kim, S.
Chattopadhyay, and C. V. Shank, Phys. Rev. Lett. 77, 4182 (1996).

[5] F. Carroll, in Proceedings of the Workshop on Medical and Biological Imaging with Novel X-Ray Beams, Idaho Accelerator Center, Pocatello, ID, 2003 (unpublished).

[6] K. Chouffani, F. Harmon, D. Wells, J. Jones, and G. Lancaster, in Proceedings of the 11th Advanced Accelerator Concept Workshop, Stony Brook, NY, 2004 (unpublished).

[7] K. Chouffani, F. Harmon, D. Wells, J. L Jones, and G. Lancaster, Laser Part. Beams (to be published).

[8] G. P. Le Sage, T. E. Cowan, R. B. Fiorito, and D. W. Rule, Phys. Rev. ST Accel. Beams 2, 122802 (1999).

[9] J.D. Jackson, Classical Electrodynamics (John Wiley \& Sons, New York, 1975), 2nd ed.

[10] M.A. Furman and M.S. Zisman, in Handbook of Accelerator Physics and Engineering, edited by A.W. Chao and M. Tigner (World Scientific, Singapore, 1999).

[11] G. F. Knoll, Radiation Detection and Measurements (John Wiley \& Sons, New York, 2000), 3rd ed.

[12] G.E.P. Box and N.R. Draper, Biometrika 52, 355 (1965).

[13] N. R. Draper and H. Smith, Applied Regression Analysis (John Wiley \& Sons, New York 1998), 3rd ed.

[14] R. B. Fiorito and D.W. Rule, in Optical Transition Radiation Beam Emittance Diagnostics, edited by $\mathrm{R}$. Shafer, AIP Conference Proceedings No. 319 (AIP, New York, 1994). 